\title{
Reply to Comment on "In vivo flow cytometry reveals a circadian rhythm of circulating tumor cells"
}

\author{
Xi Zhu' ${ }^{1}$, Yuanzhen Suo ${ }^{2,3 凶}$, Yuting Fu' ${ }^{1}$ Fuli Zhang ${ }^{1}$, Nan Ding ${ }^{1}$, Kai Pang ${ }^{4}$, Chengying Xie ${ }^{1}$, Xiaofu Weng ${ }^{1}$, Meilu Tian ${ }^{5}$, \\ Hao He $\mathbb{B}^{1 凶}$ and Xunbin Wei ${ }^{1,5,6}$
}

Dear Editor,

We thank Niedre et al. for their correspondence regarding our recent paper ${ }^{1}$. They proposed a point that the temporal distribution of circulating tumor cells (CTCs) monitored by diffuse in vivo flow cytometry in a multiple myeloma mouse model in their previous work ${ }^{2}$ might be different from our results. Niedre et al. claimed that CTC detection statistics deviated from Poisson but did not found circadian variations in CTC numbers in a multiple myeloma mouse model. They also cite another literature by Juratli. et $\mathrm{al}^{3}$, in which the authors reported that CTC numbers did not always correlate with tumor size during cancer progression. However, by establishing an orthotopic mouse model of prostate cancer and utilizing the technology in vivo flow cytometry (IVFC), we found CTCs exhibited bursting activity and daily oscillation in an orthotopic model of prostate cancer ${ }^{1}$.

We and Williams et al. ${ }^{2}$ both used IVFC to analyze fluctuations of CTC counts that were genetically fluorescently labeled and detected. Williams et al. ${ }^{2}$ found short-term dynamics of CTCs on 31 days after inoculation. The variability in CTC detection rates was higher than predicted by Poisson statistics. In our work, CTCs at different stages of cancer were analyzed and found with different distributions at early and advanced stages. At the early stage, CTCs were quite rare. The CTC occurrence deviated significantly from a Poisson process. CTCs

\footnotetext{
Correspondence: Yuanzhen Suo (suoyuanzhen@pku.edu.cn) or Hao He (haohe@sjtu.edu.cn) or Xunbin Wei (xwei01@sjtu.edu.cn) ${ }^{1}$ State Key Laboratory of Oncogenes and Related Genes, Shanghai Cancer Institute, Med-X Research Institute and School of Biomedical Engineering, Shanghai Jiao Tong University, Shanghai 200030, China

2Biomedical Pioneering Innovation Center, Peking University, Beijing 100871, China

Full list of author information is available at the end of the article
}

exhibited stochastic bursting activity at early stages, which could be the reason for the non-Poisson distribution. However, at the advanced stage of prostate cancer, the bursting activity of CTCs decreased and the occurrence of CTCs obeys Poisson statistics. This difference between our work and ref. ${ }^{2}$ may be induced by different CTC levels in different tumor models. More importantly, our major finding is the circadian variation of CTC counts.

The circadian variation of CTC counts revealed in our work is based on the orthotopic mouse model of prostate cancer, a solid tumor model. The multiple myeloma model used by Williams et $\mathrm{al}^{2}$ was a hematologic malignancy model. The microenvironments of solid tumors and hematologic cancers (e.g., extracellular matrix and immune cells) are different ${ }^{4,5}$. It is well known that the trafficking of immune cells and cytokines in the bone marrow exhibits dramatic circadian rhythm ${ }^{6-8}$. However, the immune system is severely damaged in SCID mice in the work of Williams et al. ${ }^{2}$. Considering the dissemination of malignant plasma cells is regulated by immune cells and cytokines in the bone marrow $^{6,9-11}$, we speculate the circadian release of CTCs in the bone marrow of SCID mice might be lost. It is worth noting that Paiva et al. reported circadian distribution of CTCs in patients with multiple myeloma ${ }^{12}$. This result suggests that the circadian rhythm of CTC counts varied among different species and cancers. Further studies using immune-competent mice to study the daily fluctuation of CTCs in hematological malignancies may help to elaborate on this issue.

The paper cited by Williams et al. in the Commentary reported that CTC counts did not always correlate with the primary tumor size ${ }^{3}$, which was different from most studies in mice and patients ${ }^{13-15}$. Juratli et al. observed the phenomenon about the short-term fluctuation of 
CTCs but did not find deeper insight into the statistical information $^{3}$. Our study reveals the regulation of CTC release during different stages of cancer in detail.

For the record, we initially submitted our paper to Light: Science \& Applications on 1st November 2020 before the publication of Prof. Niedre's paper. Our primary findings on the non-uniform distribution and circadian variation of CTCs had already been presented in the 14th International Conference on Photonics and Imaging in Biology and Medicine (PIBM) in 2017 and SPIE BiOS in 2020, respectively. We are happy to cite Prof. Niedre's papers in further studies.

In conclusion, according to all the analyses above, it should be noted that the tumor model types play an essential role in CTC release. In our orthotopic solid tumor model, the circadian rhythm of biological factors that can regulate tumor cell dissemination is involved to induce the daily fluctuation of CTCs. Although further studies are required to address whether the circadian rhythm of CTCs is common in all cancer types or just specific to several cancer types, evaluation of currently available data suggests that the dissemination of tumor cells in prostate cancer may be regulated by circadian rhythm.

\section{Author details}

'State Key Laboratory of Oncogenes and Related Genes, Shanghai Cancer Institute, Med-X Research Institute and School of Biomedical Engineering, Shanghai Jiao Tong University, Shanghai 200030, China. ${ }^{2}$ Biomedical Pioneering Innovation Center, Peking University, Beijing 100871, China. ${ }^{3}$ School of Life Sciences, Peking University, Beijing 100871, China. ${ }^{4}$ School of Instrument Science and Optoelectronics Engineering, Beijing Information Science \& Technology University, Beijing 100192, China. ${ }^{5}$ Biomedical Engineering Department, Peking University, Beijing 100081, China. ${ }^{6}$ Key Laboratory of Carcinogenesis and Translational Research (Ministry of Education/Beijing), Peking University Cancer Hospital \& Institute, Beijing 100142, China

\section{Conflict of interest}

The authors declare no competing interests.
Received: 16 August 2021 Accepted: 25 August 2021

Published online: 17 September 2021

\section{References}

1. Zhu, X. et al. In vivo flow cytometry reveals a circadian rhythm of circulating tumor cells. Light.: Sci. Appl. 10, 1-10 (2021).

2. Williams, A. L., Fitzgerald, J. E., Ivich, F., Sontag, E. D. \& Niedre, M. Short-term circulating tumor cell dynamics in mouse xenograft models and implications for liquid biopsy. Front. Oncol. 10, 2447 (2020).

3. Juratli, M. A. et al. Dynamic fluctuation of circulating tumor cells during cancer progression. Cancers 6, 128-142 (2014).

4. Wagner, J., Wickman, E., DeRenzo, C. \& Gottschalk, S. CAR T-cell therapy for solid tumors: bright future or dark reality? Mol. Ther. 28, 2320-2339 (2020).

5. Rafiq, S., Hackett, C. S. \& Brentjens, R. J. Engineering strategies to overcome the current roadblocks in CAR T cell therapy. Nat. Rev. Clin. Oncol. 17, 147-167 (2020).

6. He, W. et al. Circadian expression of migratory factors establishes lineagespecific signatures that guide the homing of leukocyte subsets to tissues. Immunity 49, 1175-1190 (2018). e1177.

7. Méndez-Ferrer, S., Lucas, D., Battista, M. \& Frenette, P. S. Haematopoietic stem cell release is regulated by circadian oscillations. Nature $\mathbf{4 5 2}, 442-447$ (2008).

8. Scheiermann, C. et al. Adrenergic nerves govern circadian leukocyte recruitment to tissues. Immunity 37, 290-301 (2012).

9. Noonan, K. \& Borrello, I. The immune microenvironment of myeloma. Cancer Microenviron. 4, 313-323 (2011).

10. Roccaro, A. M. et al. CXCR4 regulates extra-medullary myeloma through epithelial-mesenchymal-transition-like transcriptional activation. Cell Rep. 12, 622-635 (2015).

11. Vandyke, $\mathrm{K}$. et al. HIF-2a promotes dissemination of plasma cells in multiple myeloma by regulating CXCL12/CXCR4 and CCR1. Cancer Res. 77, 5452-5463 (2017).

12. Paiva, B. et al. Detailed characterization of multiple myeloma circulating tumor cells shows unique phenotypic, cytogenetic, functional, and circadian distribution profile. Blood 122, 3591-3598 (2013).

13. Fan, Z.-C. et al. Real-time monitoring of rare circulating hepatocellular carcinoma cells in an orthotopic model by in vivo flow cytometry assesses resection on metastasis. Cancer Res. 72, 2683-2691 (2012).

14. Netterberg, I. et al. Comparing circulating tumor cell counts with dynamic tumor size changes as predictor of overall survival: a quantitative modeling framework. Clin. Cancer Res. 26, 4892-4900 (2020).

15. Kaifi, J. T. et al. Circulating tumor cell levels are elevated in colorectal cancer patients with high tumor burden in the liver. Cancer Biol. Ther. 16, 690-698 (2015). 